\title{
José Berrios e Idenilso Bortolotto (eds.). Migración e Interculturalidad: Perspectivas Contemporáneas en el Abordaje de la Movilidad Humana. Santiago: Editorial San Pablo, 2017, 342 págs.
}

El libro Migración e Interculturalidad: Perspectivas contemporáneas en el abordaje de la movilidadhumana, coordinado por José Berríos e Idenilso Bortolotto, ambos pertenecientes a la fundación Scalabrini, es una clara orientación para las personas migrantes en Chile. En este libro, se realiza un análisis sobre el fenómeno social migratorio que ha aumentado considerablemente en nuestro país. Lo anterior queda demostrado con las cifras de extranjeros en Chile. Según los datos entregados por el Departamento de Extranjería del Ministerio del Interior,[1] la población migrante ha experimentado una aceleración progresiva. En este escenario, es posible afirmar que diversas entidades del estado de Chile no responden de manera consistente a las necesidades que requieren los migrantes, en comparación a otros países de Europa que evidencian el fenómeno social de la migración desde mucho antes y se encuentran en la ejecución de políticas públicas relacionadas con la educación y con la intervención para desarrollar la integración. El hecho de no estar preparado a nivel institucional ha generado un déficit en el marco laboral y social, considerando que es un fenómeno migratorio que se viene advirtiendo hace ya un tiempo.
En el primer capítulo, "Políticas de integración social para contextos de diversidad cultural" Carla Vidal Figueroa y Gustavo Castillo Rozas, destacan que desde laintegración es posible promover espacios óptimos para la interculturalidad. Entendiendo la interculturalidad como una importancia al reconocimiento y respeto de la diversidad social. La tarea de la sociedad es respetar aspectos que diferentes culturas nos puedan ofrecer. Las niñas y los niños en Chile no han sido educados para generar instancias de vínculos sociales, reconociendo valores sociales como la empatía; que es el primer paso para la integración social. Los autores revelan que una política social con enfoque intercultural debe ser capaz de promover el aprendizaje entre individuos, grupos, etnias y/o comunidades. Para esto, el Estado es el principal actor para la promoción de políticas al respecto. En este capítulo se menciona que el respeto de la diversidad cultural ha logrado abrir sus fronteras a la libre movilidad, otorgándoles facilidad para vivir, convivir y ser parte de una sociedad que los acoge e integra. Desde la promoción de una política es posible reeducar una sociedad que ha estado limitada por la escasa promoción y educación.

José Berrios e Idenilso Bortolotto (eds.). Migración e Interculturalidad: Perspectivas Contemporáneas en el Abordaje de la Movilidad Humana. Santiago: Editorial San Pablo, 2017, 342 págs. Anastassia Domaica Barrales.

Autoctonía. Revista de Ciencias Sociales e Historia, Vol. II, N²1, Enero-Junio 2018, 167-170 ISSN 0719-8213

DOI: http://dx.doi.org/10.23854/autoc.v2i1.81 
En el segundo capítulo, "Políticas y acciones en el área de educación que favorecen la interculturalidad" Andrea Riedemann y Carolina Stefoni analizan los procesos de adecuación al sistema escolar que viven los migrantes, una oportunidad para que el sistema educacional se involucre activamente en este fenómeno social. Desde la experiencia de la implementación de acciones y políticas en los países con mayor alto índice de recepción migratoria (Estados Unidos, Alemania, Reino Unido, España y Canadá), es posible aplicar en Chile la implementación de modelos sobre las necesidades educativas de los migrantes. Estos modelos incluirían acciones tales como: un diagnóstico previo, la formación intercultural para los facilitadores del aprendizaje, los profesores y la modificación del currículum para el aprendizaje, entre otros. Así, la promoción de la interculturalidad en la comunidad educativa se desarrollaría tanto fuera como dentro del aula, lo que implica considerarla como una tarea que podría ser aplicable en la medida en que la ciudadanía tome la real importancia que requiere este fenómeno, considerando que la educación es un eje principal al momento de promover una sociedad inclusiva, diversa e integradora.

En el tercer capítulo, "Inmigración e Interculturalidad en los medios de comunicación, representaciones y percepciones" Laura Perucho sostiene que los medios masivos de comunicación han sido una vía de representación estigmatizadora de la población migrante, a través de la repetición constante de una percepción de amenaza para la sociedad. Finalmente, se termina construyendo una sociedad que está todo el tiempo normalizando negativamente un espacio que debiese ser utilizado para integrar a la población migrante.

En el cuarto capítulo, "La infancia como migrante como un nuevo actor global", Iskra Pavez y Sonia Parella ofrecen una revisión al estado del arte sobre la infancia migrante. Se señala que los flujos migratorios se caracterizan por su alta feminización y por la realidad que enfrentan los migrantes. Estos actores participan en migraciones familiares en búsqueda de oportunidades, pero en realidad se encuentran con amenazas más que oportunidades, dado a la precariedad en la cual viven. En este capítulo se hace una especial mención a la escolarización de las niñas y niños migrantes, ya que nuevamente se logra determinar que la educación es un eje principal para poder promover políticas de integración social y educacional. La educación es un derecho y, por lo tanto, resulta importante comprender que se requiere de una comunidad educativa preparada. En los estudiantes migrantes podemos ver situaciones de fracaso escolar o de falta de expectativas, dado que no siempre se diversifica el proceso de enseñanzaaprendizaje. En consecuencia, es posible adaptarse y adaptar a estudiantes a la realidad del currículum nacional. La innovación en materia curricular, debe comprender las necesidades del nuevo alumnado.

En el quinto capítulo, "Cadenas globales de cuidado entre Chile y Bolivia y migración circular" Andrea Comelin y Sandra Leiva, académicas de la Universidad Arturo Prat, mencionan que las condiciones de frontericidad determinan que la migración circular se desarrolle en términos irregulares. Por ello, los migrantes de ambos géneros se exponen a constantes abusos laborales. En este capítulo, se hace referencia al género femenino como indicador de mayor violencia en este tipo de trabajos, constituyendo uno a puertas cerradas y con una serie de factores que expresan un índice de violencia normalizado a nivel mundial. Sin embargo, aquí encontramos un punto en el cual coinciden todos los capítulos de este libro, que en Chile, el gobierno, las instituciones que debiesen velar por el cuidado de los migrantes están atrasados en políticas en intervenciones sociales y se sigue normalizando la violencia. La propuesta del capítulo es sugerir una mejora en el marco legal con especial atención al fenómeno migratorio. 
En el sexto capítulo, "La mediación intercultural: una propuesta de intervención para la integración”, José Berrios y Viviana Vargas-Salinas proponen como eje la promoción de los procesos de participación cultural y gestión democrática de los conflictos culturales. A esto se le denomina mediación intercultural. Es posible la mediación cultural cuando existe diálogo y voluntad, para ello, es necesario abrir espacios de reflexión. Creemos que las propuestas de este capítulo podrían ser aplicables en los departamentos de convivencia en colegios, donde existen altos índices de migración, tomando esta como una alternativa educativa para la sociedad. No obstante, para que la resolución de conflictos sea válida, se debe educar a la sociedad en función de tres tipos de mediación: preventiva, rehabilitadora y creativa. La primera tiene relación con reforzar el dialogo de las personas que la implican. La segunda orientada hacia la solución del conflicto, determinando un acuerdo y la última fortalece las relaciones interpersonales.

En el séptimo capítulo "Reflexiones sobre la figura del extranjero y la cuestión de la integración desde una mirada intercultural", Claudio Bolzman plantea diferentes reflexiones sobre el fenómeno migratorio desde una perspectiva asociada a la brecha que existe en los inmigrantes percibidos como extranjeros. $\mathrm{Si}$ bien, la lectura general del capítulo invita a reflexionar sobre cómo se exponen los migrantes en una sociedad sedentaria (en contraste con una sociedad nómade), profundiza sobre las experiencias de aquellos. La sociedad tiende a suponer características de los migrantes sin conocer la realidad de estos, pero cada uno tiene una percepción diferente, las necesidades pueden repetirse en cada caso. Finalmente, el capítulo muestra una visión de lo que hay que tener en cuenta a la hora de aplicar el enfoque intercultural que está ligado a la integración de los "recién llegados".
En el octavo capítulo, "Movilidades Humanas e integración social", Norma Montesino enmarca la necesidad del trabajo social en el fenómeno migratorio post segunda guerra mundial. En este capítulo, indica que al comprender las movilidades humanas contemporáneas el trabajo social tiene y tendrá un rol importante. Ahora bien, según Benavides \& Rodas,[2] el ser humano en la condición de migrante experimenta un proceso complejo al momento de establecerse en un sitio diferente a aquel en donde ha nacido o residido, reconociéndose esto como movilidad humana. Cabe añadir a esta definición que la movilidad, además, es un derecho humano establecido en la Declaración Universal de los Derechos Humanos (1948). Consideramos que este capítulo debiese ser el primero, porque permite comprender qué es la movilidad humana en diversas culturas y países, y cómo esta se vincula con los procesos migratorios globales. Los planteamientos de este capítulo ayudan a sensibilizar al lector frente a un fenómeno que desde hace un siglo viene siendo materia de atención, expresando una necesidad social, de empatía y compromiso social. Posiblemente, a través del trabajo social reformulado e incorporando la movilidad humana se puede actuar como principio organizador de la vida social.

En el último capítulo "Investigación social cualitativa con migrantes y familias transnacionales", Dolores Frías, Laura Badenes, Héctor Monterde, Oscar García y Marcos Pascual, reconocen la importancia de entender, conocer y aprender sobre los fenómenos que se han presentado, la veracidad de los nuevos fenómenos sociales que subyacen y la importancia de las metodologías de investigación en el marco de diseñar propuestas de intervención desde la evidencia, valorando la confianza en los procesos de investigación de cada autor.

El libro Migración e interculturalidad: perspectivas 
contemporáneas en el abordaje de la movilidad humana, permite evidenciar desde diversos aspectos el fenómeno social de la migración, tanto en Chile como en otros países. Del mismo modo, la posibilidad de aplicar los avances en políticas públicas de otros países a la realidad chilena. Si bien, son diversos los temas que están interrelacionados entre sí, resulta ser un poco redundante en cuando a las diferentes perspectivas que se abordan, también es importante destacar que la lectura tiene una finalidad que promueve una educación hacia la integración y convivencia que tiene como tarea sensibilizar a la sociedad, tomando en cuenta el fenómeno como una oportunidad no como una amenaza.

\section{NOTAS}

[1] Departamento de Extranjería y Migración. Reportes Migratorios, Población Migrante en Chile. Recuperado de http://www.extranjeria.gob.cl/ noticias/2017/09/11/dem-publica-completo-reporte-sobre-la-poblacion-migrante-en-chile/

[2] Benavides, G. y Rodas, S. Definiciones básicas de movilidad humana. Recuperado de: http:// cmhecuador.blogspot.cl/2012/07/dfiniciones-basicas-sobre-la-movilidad.html

Anastassia Domaica Barrales Universidad Bernardo O'Higgins, Chile 